\title{
EDITORIAL
}

\section{Milestones in synthetic (micro)biology}

\author{
This Focus issue highlights the growth of synthetic biology as a vibrant and vigorous scientific \\ discipline that has its roots firmly grounded in microbiology.
}

Synthetic biology has been predicted to heal us, feed us and fuel us. Using the design principles and terminology of the more formal engineering disciplines, synthetic biology involves both the construction and implementation of novel genetic systems and the rewiring of natural biological systems for useful purposes, such as the generation of biofuels and pharmaceutical agents. Since its debut in 2000, the field has undergone periods of rapid growth, interspersed with periods of stagnation owing to the difficulties of engineering in the complex environment of living cells. Despite such inherent technical challenges, the first 'real-world' application was recently achieved with the entry of a semi-synthetic version of the antimalarial drug artemisinin into commercial production.

As microbiologists, we would argue that synthetic biology is firmly grounded in microbiology, with the bacterium Escherichia coli and the yeast Saccharomyces cerevisiae providing the 'nuts and bolts' for most of the key early developments, as outlined by Collins and colleagues on page 381 . The creation of synthetic genetic circuits was initially the main focus of the field and, owing to our relatively comprehensive understanding of its genome, the ease of genetic manipulation and the vast repertoire of biochemical activities it encodes, E. coli was a convenient source of biological 'parts' with which simple synthetic circuits could be engineered. In January 2000, the first such circuits were reported and comprised elegant transcriptional switches with predictable and dynamic behaviour (the toggle switch and the repressilator).

The modularity and complexity of synthetic circuits has grown considerably since then. Multiple-input logic gates, genetic clocks and quorum-sensing and lightsensing circuitry, among other devices, have been created, in addition to the development of powerful tools to modulate the behaviour and output of such constructs. As described by Qi and Arkin on page 341, synthetic noncoding RNAs, many of which are derived from bacteria (such as riboswitches and ribozymes), have emerged as a versatile class of regulators that can be used to build sophisticated synthetic biological systems. Chief among the RNA-based tools is the type II CRISPR-Cas (clustered, regularly interspaced short palindromic repeatsCRISPR-associated proteins) system from Streptococcus pyogenes, which has been adapted for both the activation and repression of gene expression. Moreover, the system has recently been developed for genome-scale editing of organisms as diverse as yeast, plants and mice, and even human cells. Other notable achievements on the genome scale include the creation of a bacterium with an entirely synthetic genome ${ }^{1}$ and the construction of a designer yeast chromosome ${ }^{2}$, which is a major advance towards building a completely synthetic eukaryotic genome.

The successes that have been achieved with E. coli and S. cerevisiae, such as the production of semi-synthetic artemisinin - as described by Paddon and Keasling on page 355 - have meant that these model microorganisms remain the mainstay as chassis organisms. However, they are often incompatible with downstream biotechnological applications, which has led de Lorenzo and colleagues, on page 368 , to advocate the use of alternative microorganisms, such as the environmental pseudomonads, which boast the physiological and metabolic traits required for industrial-scale applications. Furthermore, the choice of chassis organism is often limited by the contextdependence of biological parts, which is one of the major challenges going forward, particularly as the field embarks on the engineering of mammalian cells.

It could be argued that synthetic biology has yet to deliver on its promise to heal us, feed us and fuel us, particularly as we have only recently reached the first milestone on the path to achieving one of these lofty goals. It has been said that "the synthetic part is easy, it's the biology part that's confounding" (REF. 3), and the translation of proof-of-concept designs into real-world applications is still a major challenge. However, great progress has been made, and the field should be commended for its achievements. At the beginning of May, this Focus issue will become part of a Web Special that will merge this selection of newly commissioned articles with new content from Nature Methods and Nature, and will showcase articles that have been published by Nature Publishing Group in the past year, all of which - to us at least emphasize the central importance of microbiology to the field. With continued funding, the future certainly seems bright, and it is with great expectations that we look forward to arriving at the next major milestone.

\footnotetext{
1. Gibson, D. G. et al. Science 329, 52-56 (2010).

2. Annaluru, N. et al. Science http://dx.doi.org/10.1126/science. 1249252 (2014).

3. Gardner, T. S. et al. Trends Biotechnol. 31, 123-125 (2013)
} 